\title{
A 1.8-V Fully Differential Two-Stage CMOS OTA with 2.8V-Swing for High Speed Pipelined ADC
}

\author{
Xiaodan Zhou ${ }^{\mathrm{a}}$, Langcheng Lei ${ }^{\mathrm{b}}$, Chen Su ${ }^{\mathrm{b}}, \mathrm{Ai}$ Guo ${ }^{\mathrm{b}}$ and Dongmei Zhu ${ }^{\mathrm{b}}$ \\ Analog IC Design Center of SISC, Nanping, Chongqing, China \\ adavidaayaa@126.com, ${ }^{b}$ Raka81@126.com
}

Keywords: OTA, GBW, pipeline, ADC, CMOS.

\begin{abstract}
A low power, high dc gain, high speed and large swing operational transconductance amplifier (OTA) is proposed. The OTA achieve $104 \mathrm{~dB}$ de gain and $3.65 \mathrm{GHz}$ gain bandwidth product (GBW) and only dissipates $38 \mathrm{~mW}$ under $1.8 \mathrm{~V}$ power supply. It is fabricated in $0.18 \mathrm{um}$ CMOS process and occupies $0.146 \mathrm{~mm} 2$. It is integrated in a $14 \mathrm{~b} 50 \mathrm{MSPS}$ pipeline ADC which delivers up to $82.3 \mathrm{dBc}$ spur-free dynamic range (SFDR) and with $2.4 \mathrm{MHz}$ input tone and can be used in many communication systems where fast and precise date converting is required.
\end{abstract}

\section{Introduction}

Modern wireless communication systems have greatly driven the development of high-speed high-resolution A/D converters (ADCs) with good dynamic performance at IF, low power and cost effective. There are many ADC topologies which are suited for the wireless applications such as mobile base station or navigation system, the most popular one for them is pipeline ADC [1].

Traditional pipeline ADC always has a Multiplying DAC (MDAC) in each pipeline stage which contains a high GBW OTA together with charge redistribution DAC [2]. It can achieve high speed and provide excellent linearity excpt with more power consumption. Recently, there are new type pipeline ADCs which have combined the advantages of both pipeline and SAR structure to achieve low power [3]. However, the new topology can only satify medium resolution and always have digital calibration which increase the complexity of ADC. Hence, for pipeline ADC with high resolution, the traditional structure is more preferred than new one.

As the core part of the MDAC, the OTA is the most difficulty one to design, especially at high speed and resolution which requires more GBW [4]. Although the CMOS amplifier has been invented since 1960s and has been developped greatly over the past 30 years, it is still a challenge to design two or more stages within an amplifier because of the complexity of analyzing the poles and zeros and done the compensation. Therefore, trick and trade-off must be taken to achieve the goal.

This paper describes the implement of a high gain and high speed OTA with great swing, which is very suitable for pipeline ADCs used in communication systems where low power consumption is often required. This paper is divided into five main parts. After the amplifier architecture have been decided in Section II, Section III describes the circuit techniques that were used to achieve high gain and high speed while maintaining low power consumption. The performance result is presented in Section IV and Section V concludes the paper.

\section{Architecture Considerations}

\subsection{Section Headings.}

The design target is a high GBW CMOS OTA with low power consumption which can be used in 14 16bits pipeline ADC up to 150MSPS sample rate. There are many amplifier topologies can be used to implement high GBW amplifier, such as telescopic, folded-cascode, two-stage or gain-boosted and so on, which can be seen in the table I. 
Table 1 Amplifier Structure Summary

\begin{tabular}{cccccc}
\hline Topology & Gain & Output & Speed & $\begin{array}{c}\text { Power } \\
\text { Dissipation }\end{array}$ & \multirow{2}{*}{ Noise } \\
\hline Telescopic & Medium & Medium & Highest & Low & Low \\
Fold-Cascode & Medium & Medium & High & Medium & Medium \\
Two-Stage & High & Highest & Low & Medium & Low \\
Gain-Boosted & High & Medium & Medium & High & Medium \\
\hline
\end{tabular}

As the OTA must achieve $2.8 \mathrm{~V}$ swing with $1.8 \mathrm{~V}$ supply voltage, the two-stage amplifier is the only choice for it. However, for large dc gain, the traditional two-stage amplifier can only provide $60-70 \mathrm{~dB}$ while a 16bit ADC often requires more than $100 \mathrm{~dB}$. Therefore, change must be made to the well-known two-stage amplifier.

For the two-stage amplifier, the first stage of it often provides the main gain while the second stage provides high swing and driving force. To achieve high gain, the common five transistors which comprise the first stage can be placed with telescopic amplifer for its gain, power dissipation and noise advantage. And with telescopic, the gain-boosted can be used simultaneously naturally.

The second stage of the two-stage amplifier is usually a common source amplifier, which can provide the largest swing and enough driving force, and it can be used without change.

The more important part of the two-stage amplifier is its compensation. However, as many research have been spent on two-stage compensation, it will not be a big challenge.

As a result, a two-stage amplifier which has a telescopic amplifier with gain-boosted first stage, and a common second stage with miller compensation will be adopted to implement the OTA, as can be seen in Fig.1.

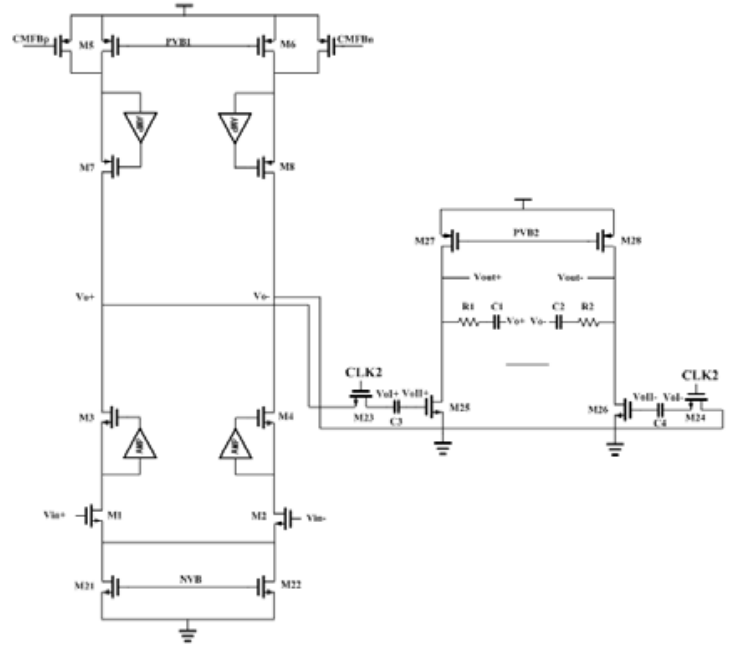

Fig. 1 The two-stage amplifier architecture

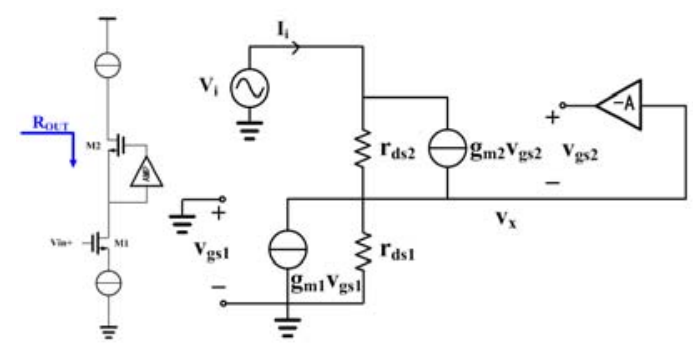

Fig. 2 Gain-boosted equivalent schematic

\section{Circuit Implementation}

\subsection{The first stage amplifier.}

The first stage of the two-stage amplifier is a telescopic with gain-boosted amplifier. With telescopic, in the signal path there will be all NMOS transistors, while there are PMOS transistors in fold-cascode amplifier. As the NMOS transistor usually has greater mobility of carriers then the PMOS counterparter, the telescopic has greater gain and lower noise than the fold-cascode structure.

For the gain-boosted amplifier, the increase of the gain will be relative to the output impedance increased. Fig. 2 shows the schematic of the gain-boosted and the equivalent small signal model.

The output impedance of the cascode with gain-boosted is as in (1)

$R_{\text {out }}=\frac{V_{i}}{I_{i}} \approx(1+A) \cdot g_{m 2} \cdot r_{d s 2} \cdot r_{d s 1}$

For the gain-boosted amplifier, it can be implemented with simply single common source amplifier or fully differential structure. With fully differential, there must be a additional 
common-mode feedback circuit to stabilize the output common-mode voltage of the gain-boosted. This will increase power dissipation and design complexity. For simplicity, the gain-boosted is composed of single common source amplifier to eliminate the need for common-mode feedback circuit, and has cascode load instead of a single PMOS load, as can be seen them in Fig.3 which shows the whole circuit.

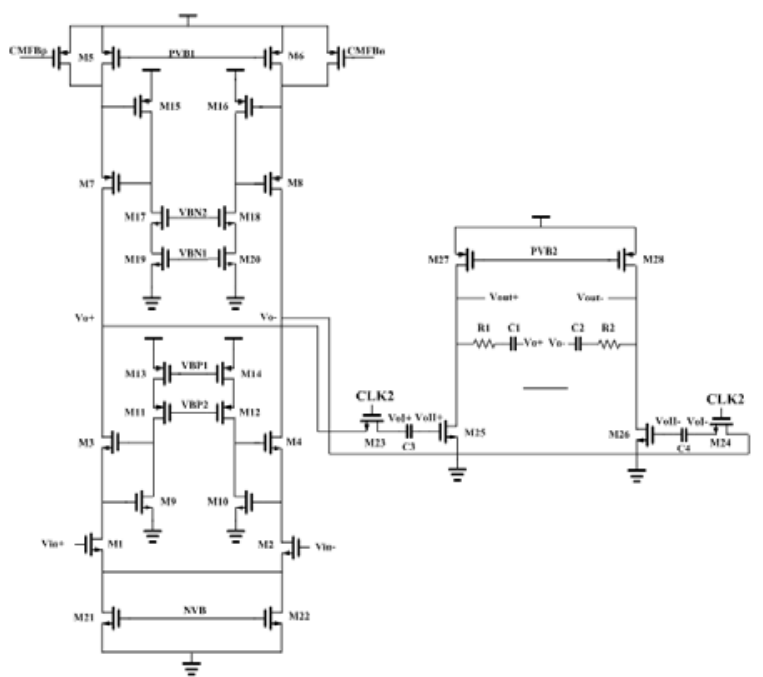

Fig. 3 The entire amplifier with gain-boosted

For gain-boosted amplifier, its small gain is given by (2) and (3) for NMOS input and PMOS input respectively.

$$
\begin{aligned}
& g_{m 9} \square\left(r_{d s 9} \square g_{m 10} \square r_{d s 10} \square r_{d s 13}\right) \\
& g_{m 15} \square\left(r_{d s 15} \square g_{m 17} \square r_{d s 17} \square r_{d s 19}\right)
\end{aligned}
$$

And the gain of the first stage is as in (4)

$$
A_{v 1}=g_{m 1} \cdot\left[\begin{array}{l}
\left(1+g_{m 9} \cdot\left(r_{d s 9} \square g_{m 11} \cdot r_{d 11} \cdot r_{d s 13}\right)\right) \cdot g_{m 3} \cdot r_{d s 3} \cdot r_{d s 1} \square \\
\left(1+g_{m 15} \cdot\left(r_{d s 15} \square g_{m 17} \cdot r_{d s 17} \cdot r_{d s 19}\right)\right) \cdot g_{m 7} \cdot r_{d s 7} \cdot\left(r_{d s 5} \square r_{d s c m b l}\right)
\end{array}\right]
$$

\subsection{The second stage.}

The second stage of the amplifier is a simply common source amplifier, and its dc gain is given by (5)

$$
A_{v 2}=g_{m 25} \cdot\left(r_{d s 25} \square r_{d s 27}\right)
$$

Hence, the overall dc gain of the two-stage amplifier is the product of stage one and two, as is given by (6)

$$
\begin{aligned}
A_{v}= & A_{v 1} \cdot A_{v 2} \\
= & g_{m 1} \cdot\left[\begin{array}{l}
\left(1+g_{m 9} \cdot\left(r_{d s 9} \square g_{m 11} \cdot r_{d s 11} \cdot r_{d s 13}\right)\right) \cdot g_{m 3} \cdot r_{d s 3} \cdot r_{d s 1} \square \\
\left(1+g_{m 15} \cdot\left(r_{d 15} \square g_{m 17} \cdot r_{d s 17} \cdot r_{d s 19}\right)\right) \cdot g_{m 7} \cdot r_{d s 7} \cdot\left(r_{d s 5} \square r_{d s c m b b}\right)
\end{array}\right] . \\
& g_{m 25} \cdot\left(r_{d s 25} \square r_{d s 27}\right)
\end{aligned}
$$

As it is dc-couple between the first and the second stage, and the output common-mode voltage of the first stage is not equal the input common-mode voltage of the second stage, there must be a voltage shifter for the second stage. As can be seen in Fig.3, the capacitance of $\mathrm{C}_{3}$ and $\mathrm{C}_{4}$, NMOS switch of $\mathrm{M}_{23}$ and $\mathrm{M}_{24}$ with clock $\mathrm{CLK}_{2}$ serve as the shift.

\subsection{Frequency response of amplifier}

In midband frequencies, the gain is as seen in (7)

$$
\begin{aligned}
A_{V}(s) & =A_{V 1} \cdot A_{V 2} \\
& \cong A_{V 2} \cdot \frac{g_{m 1}}{s C_{1} A_{v 2}} \\
& =\frac{g_{m 1}}{s C_{1}}
\end{aligned}
$$

And the unity-gain frequency is given in (8), ignoring the influence of gain-boosted and common-mode feedback circuit. 
$\omega_{t a}=\frac{g_{m 1}}{C_{1}}$

In Fig.4 it shows the equivalent small circuit of the amplifier, ignoring the influence of gain-boosted and common-mode feedback circuit, as they are not in the signal path.

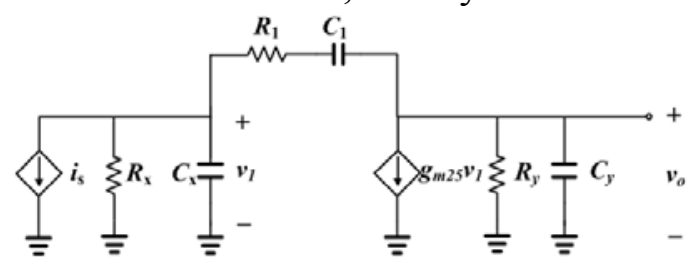

Fig. 4 Small signal equivalent of amplifier

It can be deduced from the equivalent circuit the transfer function as can be seen in (9) and (10)

$\frac{v_{o}}{v_{i n}}=\frac{g_{m 1} R_{x} R_{y}\left[1-s C_{1}\left(\frac{1}{g_{m 25}}-R_{1}\right)\right]}{1+b s+c s^{2}+d s^{3}}$

Where b, c, d are as in (10)

$b=R_{y}\left(C_{y}+C_{1}\right)+R_{x}\left(C_{x}+C_{1}\right)+R_{1} C_{1}+g_{m 1} R_{x} R_{y} C_{1}$

$c=R_{x} R_{y}\left(C_{x} C_{y}+C_{1} C_{x}+C_{1} C_{y}\right)+R_{1} C_{1}\left(R_{x} C_{x}+R_{y} C_{y}\right)$

$d=R_{x} R_{y} R_{1} C_{x} C_{y} C_{1}$

Where $R_{x}, C_{x}, R_{y}, C_{y}$ and $i_{s}$ is as in (11)

$R_{X}=\left[\begin{array}{l}\left(1+g_{m 9} \cdot\left(r_{d s 9} \square g_{m 11} \cdot r_{d s 11} \cdot r_{d s 13}\right)\right) \cdot g_{m 3} \cdot r_{d s s} \cdot r_{d s 1} \square \\ \left(1+g_{m 15} \cdot\left(r_{d s 15} \square g_{m 17} \cdot r_{d s 17} \cdot r_{d s 19}\right)\right) \cdot g_{m 7} \cdot r_{d s 7} \cdot\left(r_{d s 5} \square r_{d s c m \mid b}\right)\end{array}\right]$

$C_{X} \cong C_{d b 3}+C_{d b 7}+C_{g 525}$

$R_{y}=r_{d 225} \square r_{\text {d } 27}$

$C_{y} \cong C_{d b 25}+C_{d b 27}+C_{L}$

$i_{s}=g_{m 1} \cdot v_{\text {in }}$

Then it can be concluded that the poles and zero are as in (12) and (13).

$$
\begin{aligned}
& p_{1} \approx-\frac{1}{g_{m 25} R_{x} R_{y} C_{1}} \\
& p_{2} \approx-\frac{g_{m 25} C_{1}}{C_{x} C_{y}+C_{1}\left(C_{x}+C_{y}\right)} \approx-\frac{g_{m 25}}{C_{x}+C_{y}} \\
& p_{3} \approx-\frac{1}{R_{1} C_{x}} \\
& Z=\frac{1}{\left(\frac{1}{g_{m 25}}-R_{1}\right) C_{1}}
\end{aligned}
$$

As can be seen from above, the dominant pole is determined by miller capacitance, and the first non-dominant pole is determined by output node. The second non-dominant is at the source of cascode of the first stage and is usually at very large frequency which can be ignored.

For gain-boosted amplifier, it introduces their own poles and zeros which is so-called doublet. The doublet will affect the amplifier settling behavior and must push to higher frequency.

For good roll-off of frequency response, the speed of the gain-boosted must not be too large, especially that there is also a closed-loop between the gain-boosted and cascode transistor. As can be seen in Fig.5, with the frequency increasing, the output impedances of whole amplifier and gain-boosted are simultaneously decreasing [5]. And there are two poles in gain-boosted amplifier, where one is at output, and another is at the source of cascode transistor. 


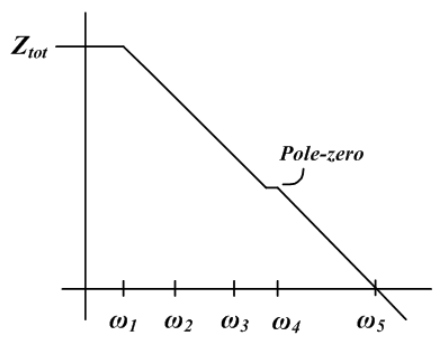

Fig.5 Output impedance with frequency increasing

Accounting for doublet, the step response is as in (14)[6]

$$
y(t)=\frac{A_{0}}{1+A_{0}}\left[1-\left(1-\frac{\omega_{p 2}}{\omega_{z}}\right) e^{-\omega_{p 2} t}-e^{-\left(A_{0}+1\right) \omega_{p} t}\right] u(t)
$$

If the pole-zero pair of $\omega_{z}$ and $\omega_{\mathrm{p} 1}$ will not cancel out each other, there will introduce an exponent term as in (15)

$$
\left(1-\frac{\omega_{p 2}}{\omega_{z}}\right) e^{-\omega_{p 2} t}
$$

To avoid the settling degeneration, the doublet must be push to the frequency between $-3 \mathrm{~dB}$ frequency of closed-loop and the non-dominant as in (16)

$$
\beta \omega_{5}<\omega_{4}<\omega_{6}
$$

\section{Measured results}

This amplifier is used in a pipeline ADC which is fabricated in a $0.18-\mu \mathrm{m}$ CMOS process. Fig 6 shows OTA used in the first stage of this pipeline ADC photomicrograph, with a total die area of $0.146 \mathrm{~mm}^{2}$.

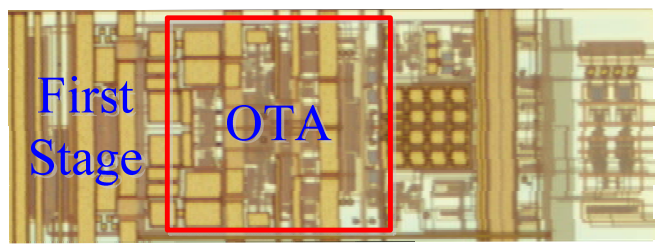

Fig.6 Pipeline ADC core photomicrograph

Table II summarizes the measure results of the ADC at sampling rate $f_{S}=50 \mathrm{MS} / \mathrm{s}$ and analog input signal $\mathrm{f}_{\mathrm{IN}}=2.4 \mathrm{MHz}$. Fig7 shows the result of INL and DNL. Fig. 8 indicates FFT curves of the ADC measured at $\mathrm{f}_{\mathrm{IN}}=2.4 \mathrm{MHz}$.

Table 2 Amplifier and ADC Performance Summary

\begin{tabular}{cc}
\hline Technology & $0.18 \mathrm{um}$ CMOS process \\
\hline VDD & $1.8 \mathrm{~V}$ \\
Input voltage range & $1 \mathrm{Vpp}$ \\
Sample rate & $50 \mathrm{MS} / \mathrm{s}$ \\
Swing & $2.8 \mathrm{~V}$ \\
DC gain & $104 \mathrm{~dB}$ \\
GBW & $3.65 \mathrm{GHz}$ \\
Phase Margin & $76 \mathrm{degree}$ \\
Power & $38 \mathrm{~mW}$ \\
OTA area & $0.146 \mathrm{~mm} 2$ \\
INL of ADC & $2.5 \mathrm{LSB}$ \\
DNL of ADC & $0.8 \mathrm{LSB}$ \\
SFDR @ fIN=2.4MHz of ADC & $82.3 \mathrm{dBc}$ \\
\hline
\end{tabular}



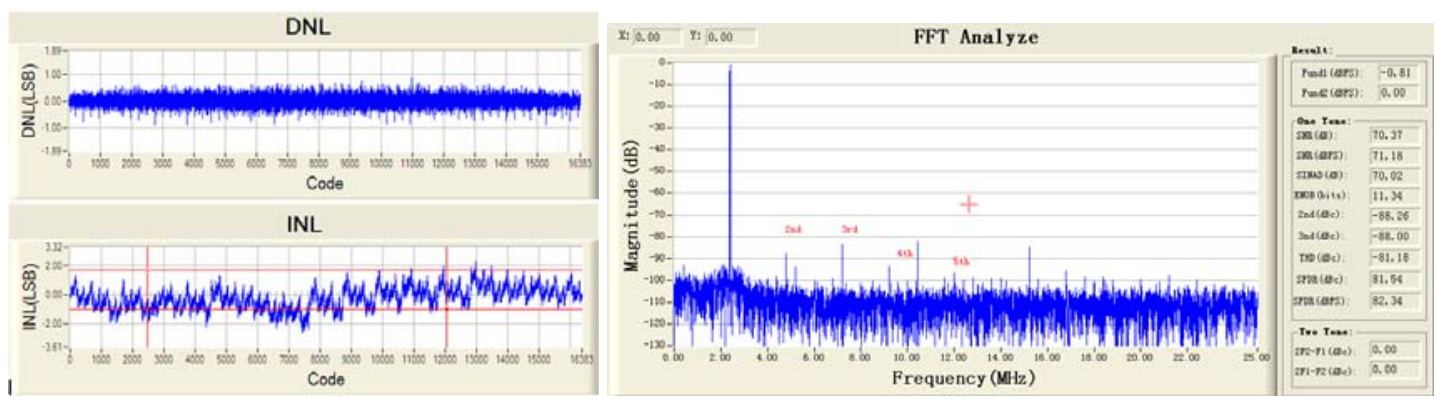

Fig.7 Measured Result

\section{Summary}

In this paper, a high GBW OTA was used in a $14-\mathrm{b}$ pipeline ADC was fabricated in a $0.18-\mu \mathrm{m}$ CMOS process. This OTA is only consumes dissipates $40 \mathrm{~mW}$ under $1.8 \mathrm{~V}$ power supply, while making the ADC achieve $82.3 \mathrm{dBc}$ SFDR with $2.4 \mathrm{MHz}$ input, which means that it is very suitable for high speed high resolution pipeline ADC requiring low power.

\section{References}

[1]. S. H. Lewis, H . S. Fetterman, G . F. Gross, J . R. Ramachandran, and T .R . Viswanathan, "10-b 20-Msamplds analog-to-digital converter,” IEEE J. Solid-State Circuits, vol. 27, pp. 351-358, Mar. 1992.

[2]. T. B. Cho and P.R. Gray, “A 10 b, 20 Msample/s, 35 mW Pipeline A/D Converter," IEEE J. Solid-State Circuits, vol. 30, pp. 166-172, Mar. 1995.

[3]. C. P. Hurrell, C. Lyden, D. Laing, D. Hummerston, and M. Vickery, "An 18 b 12.5 MS/s ADC with 93 dB SNR," IEEE International Journal of Solid State Circuits, vol. 45, no. 12, pp. 2647-2654, 2010.

[4]. W.Yang, D.Kelly, I.Mehr, M.Sayuk, and L.Singer, "A 3-V 340-mW 14-b 75-Msample/s CMOS ADC With 85-dB SFDR at Nyquist Input," IEEE J. Solid-State Circuits, vol. 36, pp. 1931-1936, Dec. 2001.

[5]. K.Bult and G.J.G.H. Geelen. "A Fast-Settling CMOS Operational Amplifier for SC Circuits with 90-dB DC Gain”, IEEE J. Solid-State Circuits, vol. 25, pp. 1379-1384, Dec. 1990.

[6]. B.Y.Kamath, R.G.Meyer, and P.R.Gray. "Relatonship between Frequency Response and Settling Time of Operational Amplifiers", IEEE J. Solid-State Circuits, vol. 9, pp. 347-352, Dec. 1974. 